\title{
Cross-Polar Aircraft Trajectory Optimization and the Potential Climate Impact
}

Banavar Sridhar and Neil Chen NASA Ames Research Center

Moffett Field, CA, USA
Hok $\mathrm{Ng}$

University of California Santa Cruz, CA, USA

Second ACCRI Symposium

Washington, DC

December 13-15 
NASA Administrator's

Office

Research Mission

Directorates
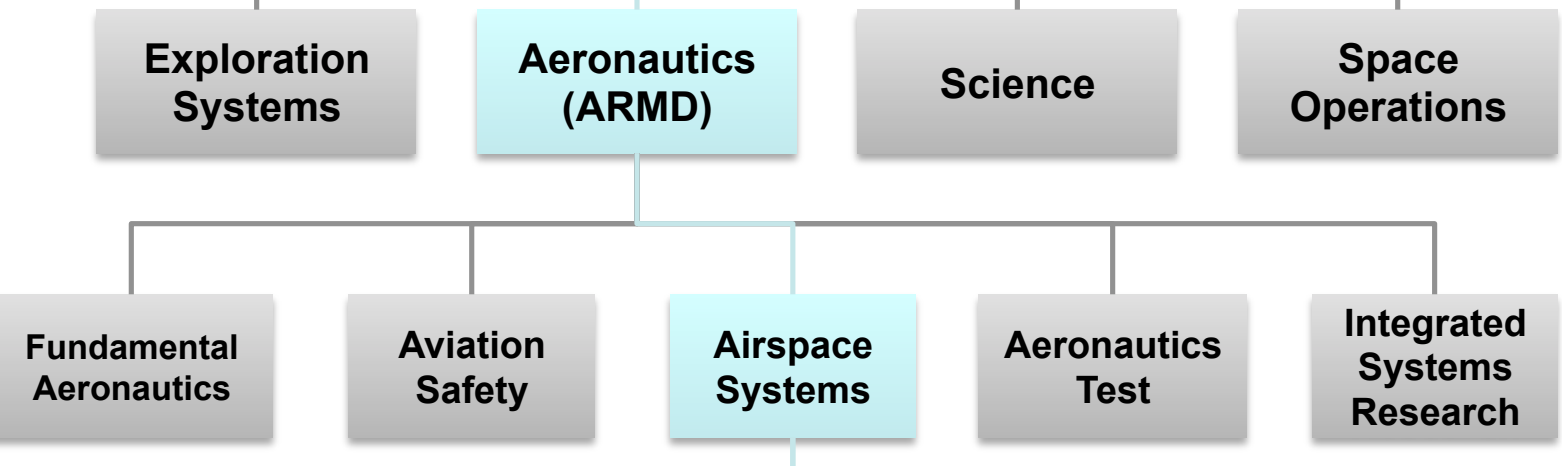

Programs

Concepts \&

Technology

Development

Systems Analysis,

Integration, \&

Projects

(CTD)

Evaluation (SAIE)

Research
Focus
Areas (RFA)

\begin{tabular}{|c|c|c|}
\hline $\begin{array}{l}\text { Separation } \\
\text { Assurance } \\
\text { (SA) }\end{array}$ & $\begin{array}{c}\text { Super- } \\
\text { Density } \\
\text { Operations } \\
\text { (SDO) }\end{array}$ & $\begin{array}{c}\text { Safe \& } \\
\text { Efficient } \\
\text { Surface } \\
\text { Operations } \\
\text { (SESO) }\end{array}$ \\
\hline
\end{tabular}

Traffic Flow

Management (TFM)
Dynamic
Airspace
Configuration

(DAC)
System \&

Portfolio

Analysis

(SPA) 


\section{Research Challenges for NextGen The two big questions}

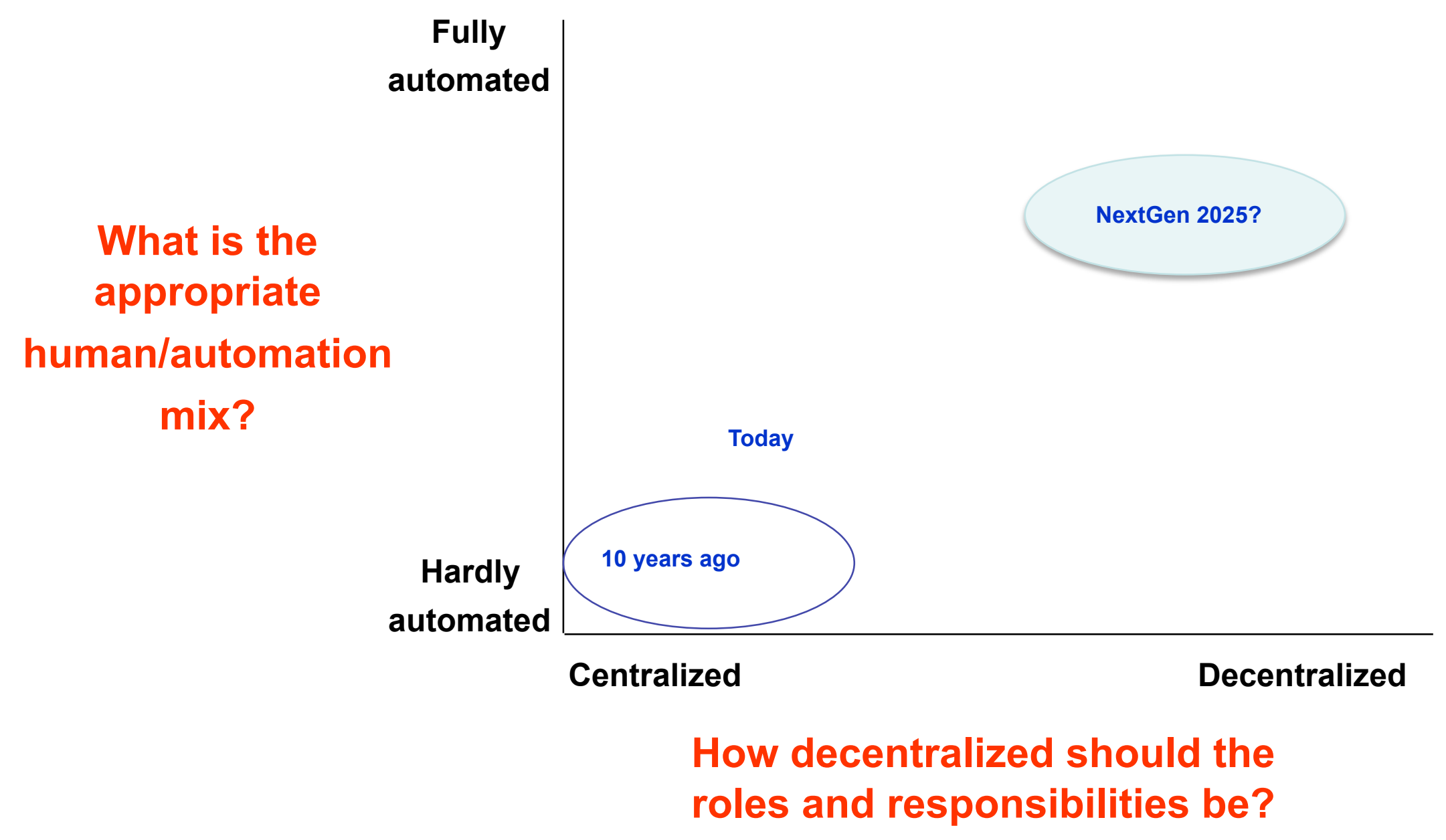




\section{Air Traffic Operations}

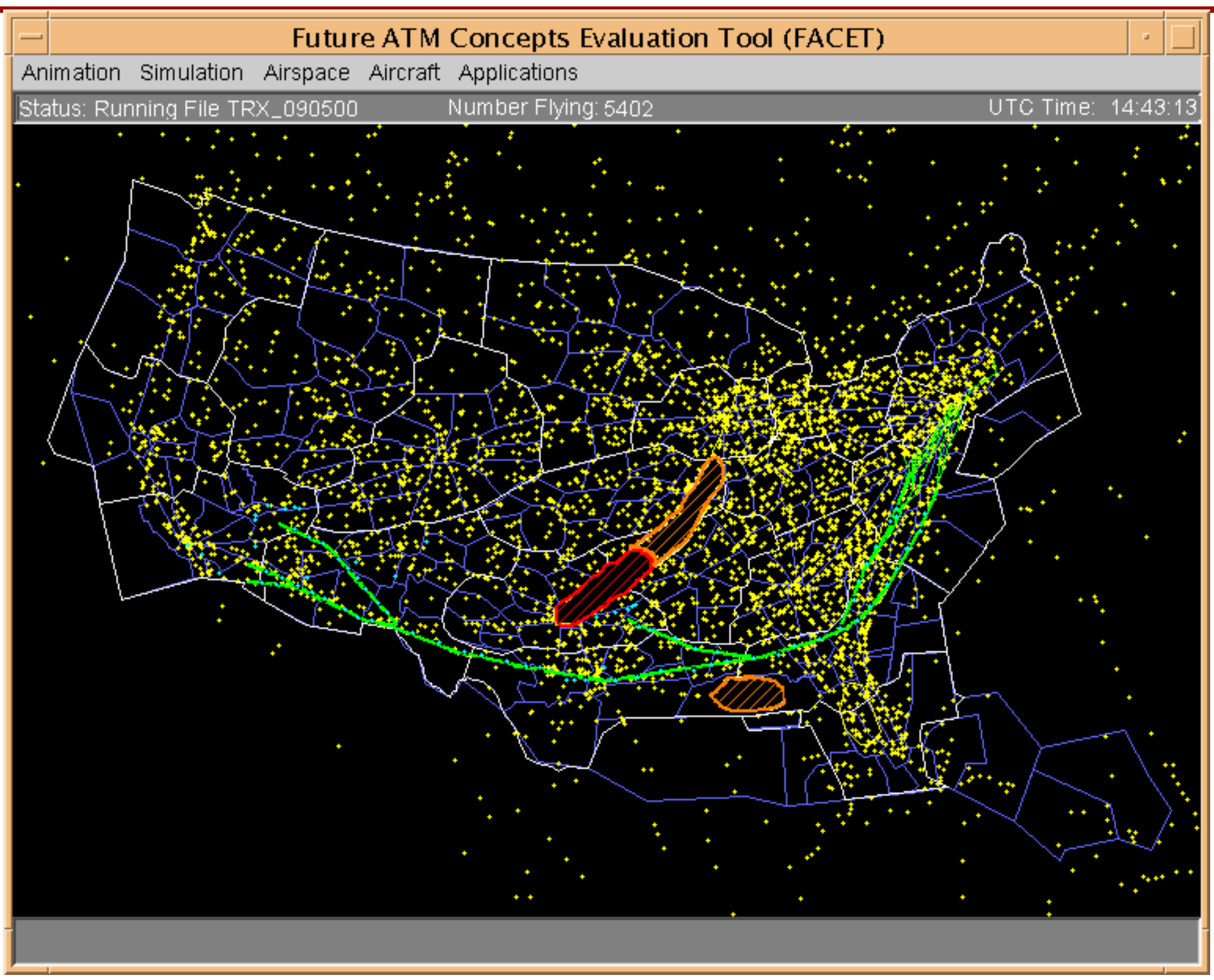




\section{Traffic Flow Management}

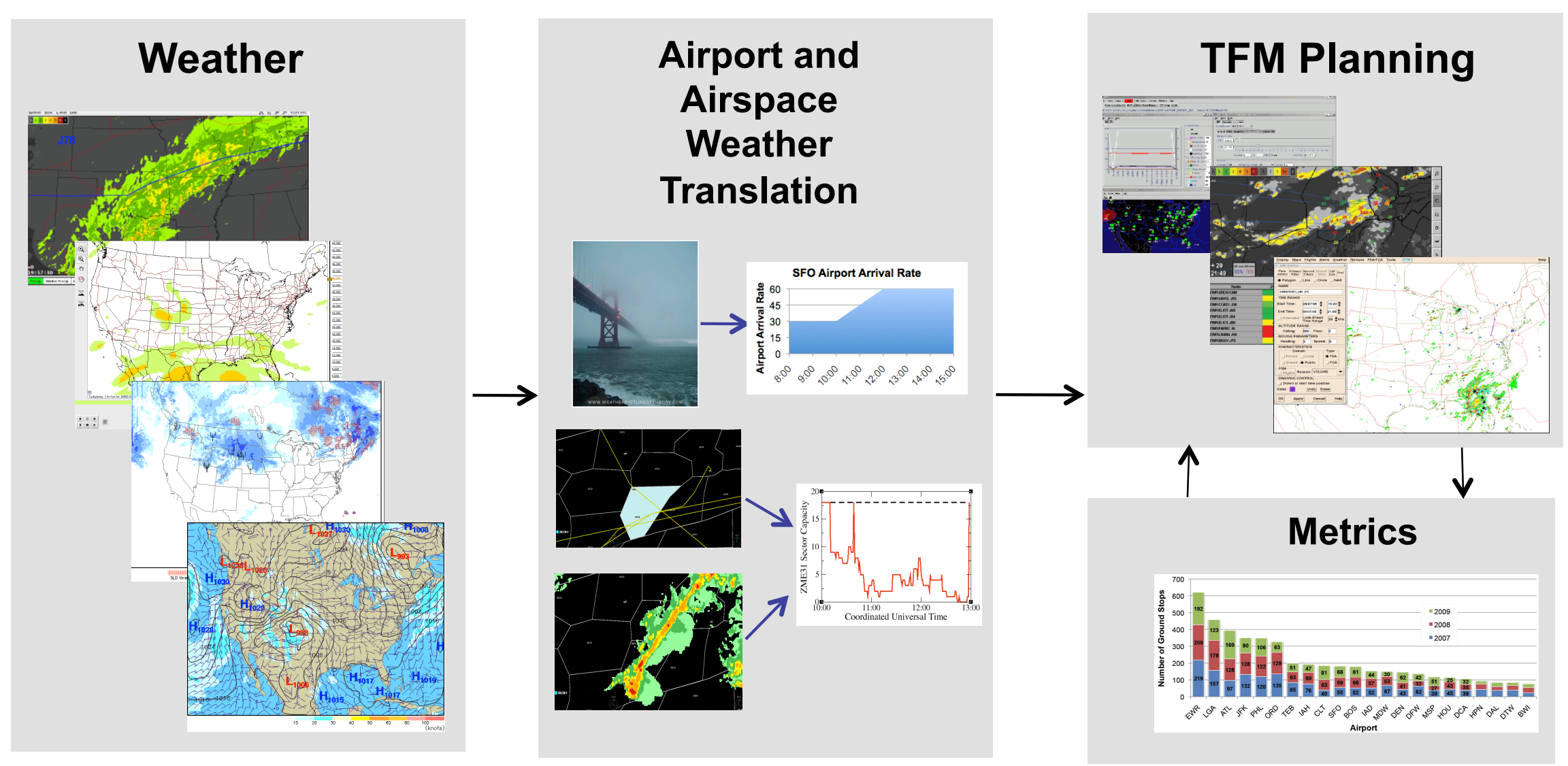

Investigate modeling, simulation and optimization techniques to manage air traffic flows subject to airspace and airport capacity constraints while accommodating user preferences with increased traffic demand in the presence of uncertainty 


\section{Current Research Goals}

- Develop optimal aircraft trajectories

- Aircraft Dynamic Models (Point Mass, 3-DOF)

- Winds, Uncertainties

- Minimize

- Fuel burn

- Climate impact of emissions

- Global Warming Potential (GWP), Global Temperature Potential (GTP)

- Persistent contrails

- Global models for contrail formation using weather forecast

- Methods based on calculus of variations

- Accuracy versus computation time

- Model convective weather and other weather hazards and translate their impact on airspace capacity

- Integrate climate models and metrics with US national airspace simulation 


\section{Cross-Polar Operation}

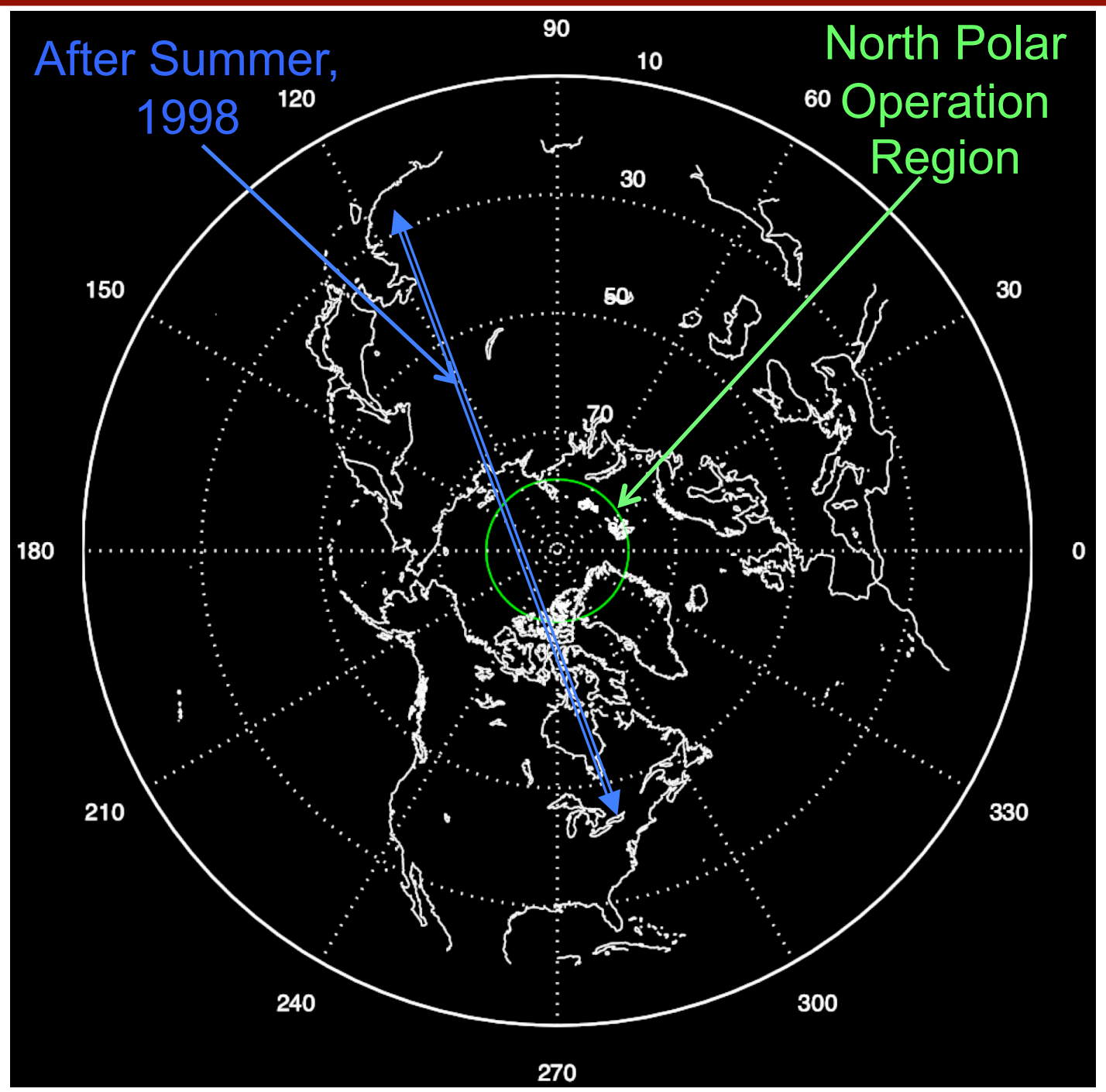

Annual operations grew from 402 flights in 2000 to 8527 flights in 2009 


\section{Current Cross-Polar Flights}

- Provide shorter paths between many North American and Asian cities

- Have not fully utilized the potential benefits of flying wind-optimal routes due to limited track flexibility

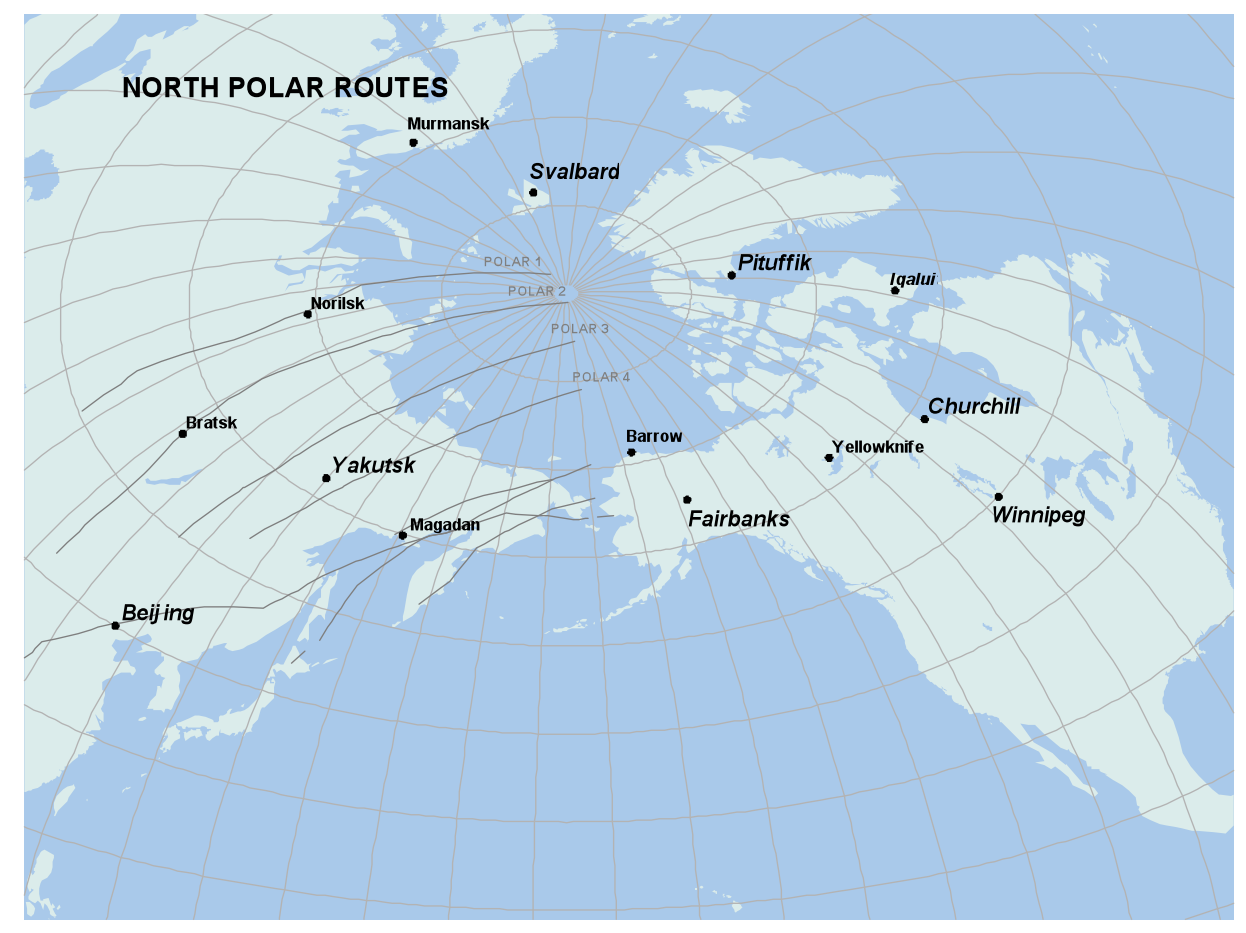

- Do not consider climate impact 


\section{Environmentally Responsible Trajectory}

- Find the optimal trajectory given the arrival and departure airports, cruise speed and winds subject to environmental constraints

- The aircraft equations of motion at a constant altitude above the spherical Earth's surface are

$$
\begin{aligned}
& \dot{\phi}=\frac{V \cos \psi+u(\phi, \theta, h)}{R \cos \theta} \\
& \dot{\theta}=\frac{V \sin \psi+v(\phi, \theta, h)}{R} \quad \text { subject to } \\
& T h=D \\
& L=W \\
& \dot{m}=-f \\
& R>h
\end{aligned}
$$




\section{Optimization Subject to Environmental Constraints}

- Optimize horizontal trajectory by determining the heading angle that minimizes the cost function

$$
\begin{aligned}
& J(h)=\int_{t_{0}}^{t_{f}}\left[C_{t}^{\leftarrow}+K(\phi, \theta, h)\right] d t \\
& K(\phi, \theta, h)=\sum_{i} C_{i} \cdot G W P_{i}(\phi, \theta, h) \cdot E I_{i} \cdot f(h)+C_{r} r(\phi, \theta, h)
\end{aligned}
$$

-Solution reduces to solving

$$
\begin{aligned}
& \dot{\phi}=\frac{V \cos \psi+u(\phi, \theta, h)}{R \cos \theta} \\
& \dot{\theta}=\frac{V \sin \psi+v(\phi, \theta, h)}{R} \\
& \dot{\psi}=\frac{-\left[F_{\text {wind }}(\psi, \phi, \theta, u, v)+F_{\text {climate }}(\psi, \phi, \theta, u, v, K(\phi, \theta, h))\right]}{R \cos \theta\left(C_{t}+K(\phi, \theta, h)\right)}
\end{aligned}
$$




\section{Atmospheric Model}

- Compute and predict contrails using Global Forecasting System (GFS)

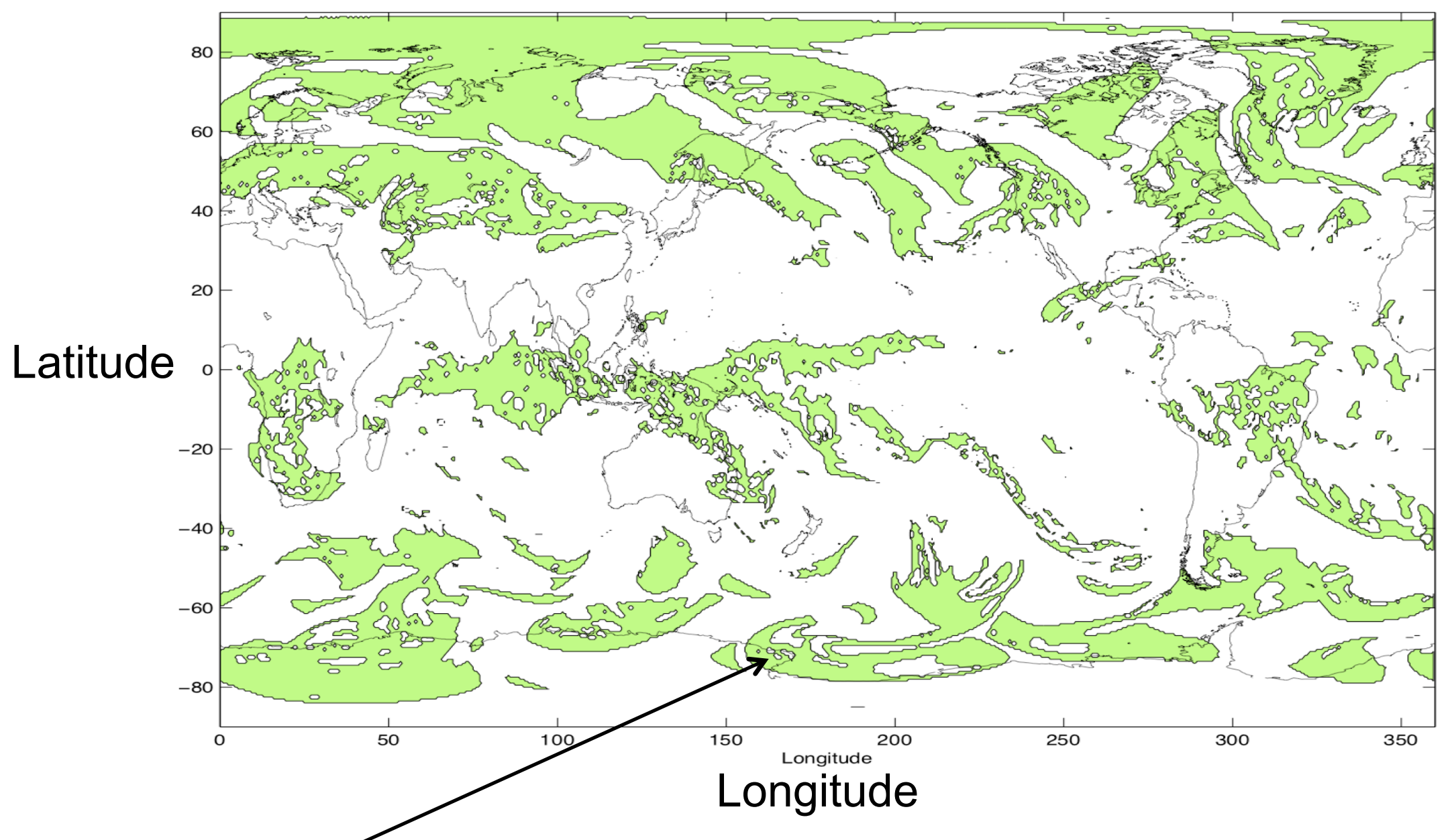

Contrail Favorable Regions at 8 p.m. EDT on Dec. 31, 2009 


\section{Fuel and Emission Models}

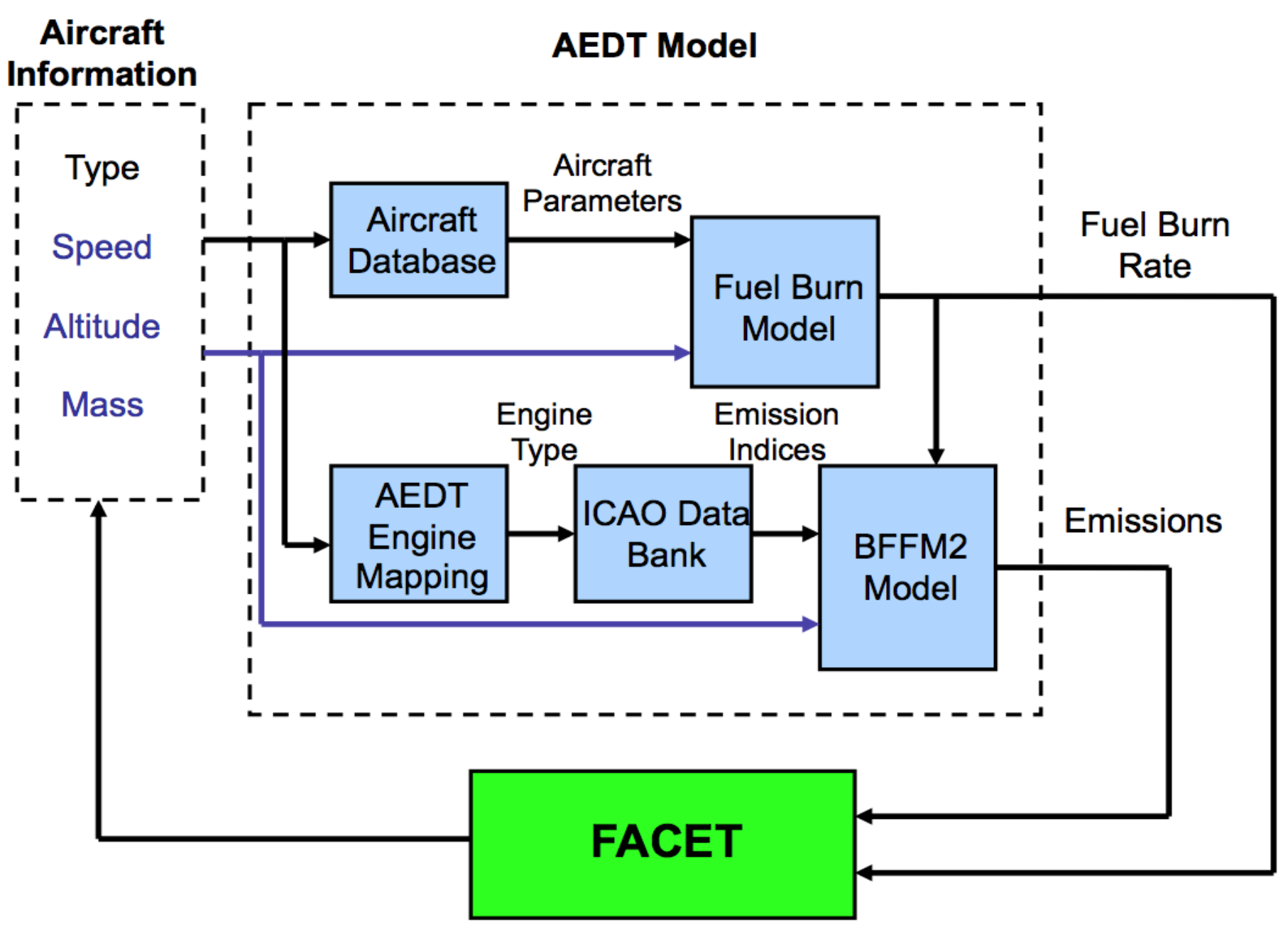

Aviation Environmental Design Tool (AEDT) Eurocontrol's Base of Aircraft Data (BADA) 


\section{Variation of Emissions with Altitude}

$$
\begin{aligned}
& e\left(\mathrm{CO}_{2}\right)=3155 \times \sigma \\
& e\left(\mathrm{H}_{2} \mathrm{O}\right)=1237 \times \sigma \\
& e\left(\mathrm{SO}_{2}\right)=0.8 \times \sigma
\end{aligned}
$$

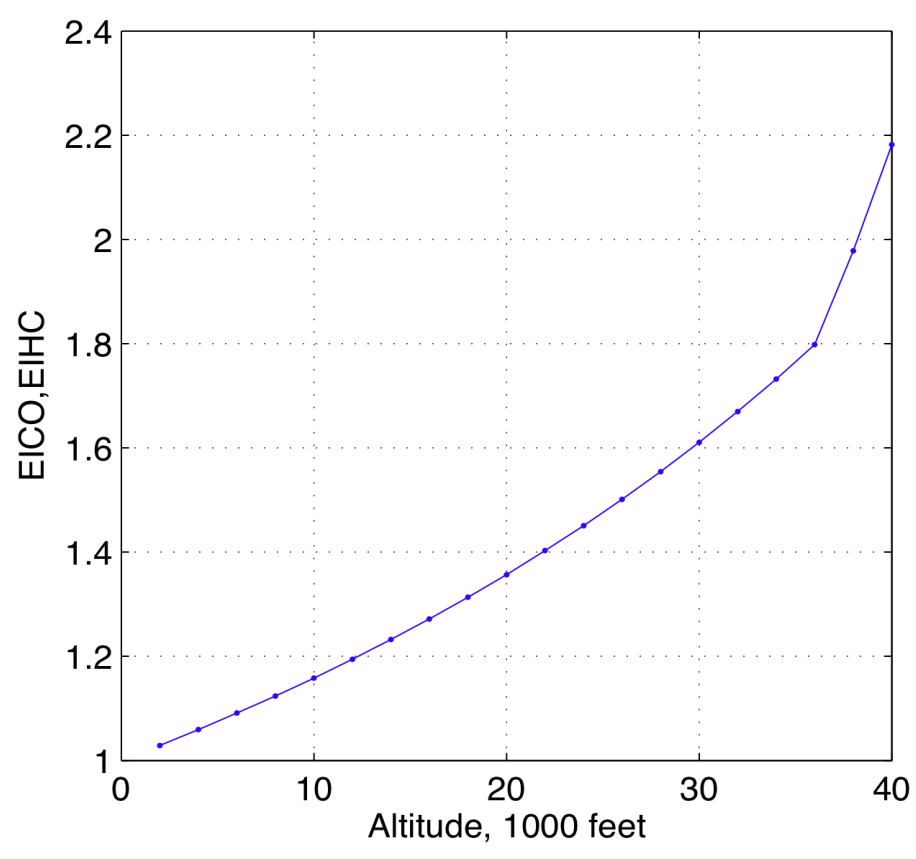

$$
\begin{aligned}
e(H C) & =E I H C \times \sigma \\
e(C O) & =E I C O \times \sigma \\
e\left(N O_{x}\right) & =E I N O_{x} \times \sigma
\end{aligned}
$$

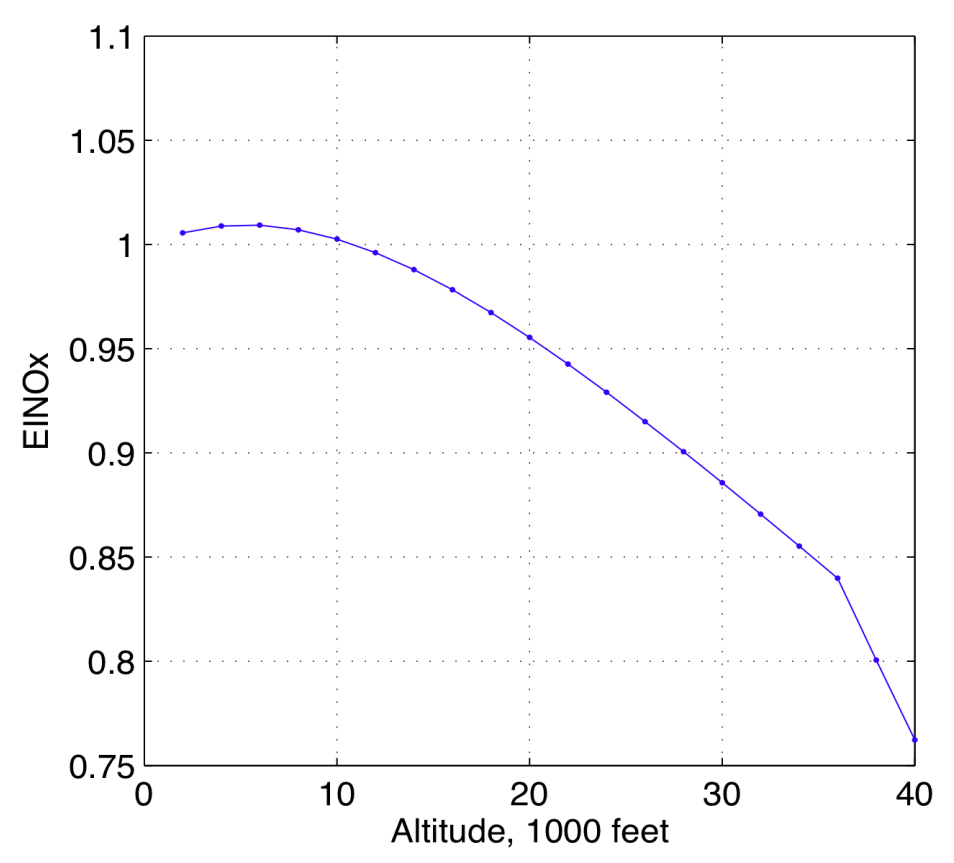

- Fuel and emission models undergoing additional verification using AEDT (Collaboration with Volpe National Transportation Systems Center) 


\section{Cross-Polar Trajectories-Chicago to Hong Kong}

Altitude at $32,000 \mathrm{ft}$

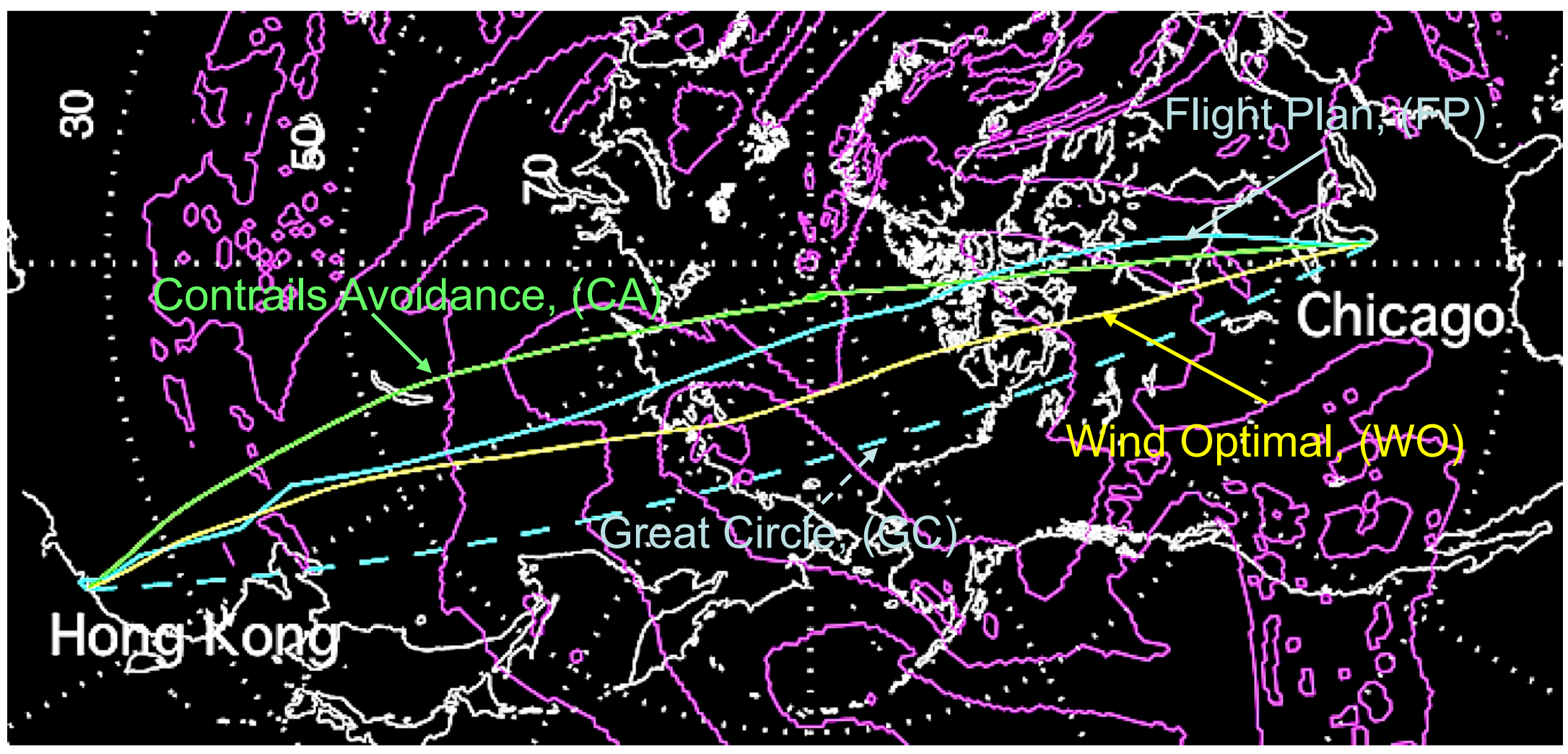




\section{Trajectories for 15 Origin-Destination Pairs}

- For 15 Origin-Destination pairs during the days with medium and high contrail formation in 2010

- Analyze the fuel burn and climate impact

- Investigate the tradeoff between persistent contrails formation and additional fuel burn, with and without altitude optimization

\begin{tabular}{|c|c|}
\hline Chicago O'Hare & $\begin{array}{l}\text { Beijing Capital, Hong Kong, Shanghai Pudong, } \\
\text { Seoul Incheon }\end{array}$ \\
\hline Hartsfield-Jackson Atlanta & Seoul Incheon \\
\hline John F. Kennedy & Beijing Capital , Hong Kong, Seoul Incheon , \\
\hline Los Angeles & Dubai \\
\hline Newark Liberty & Hong Kong, Shanghai Pudong , Singapore Changi \\
\hline Washington Dulles & Beijing Capital, Narita, Seoul Incheon \\
\hline
\end{tabular}




\section{Potential Contrails Formation in 2010}

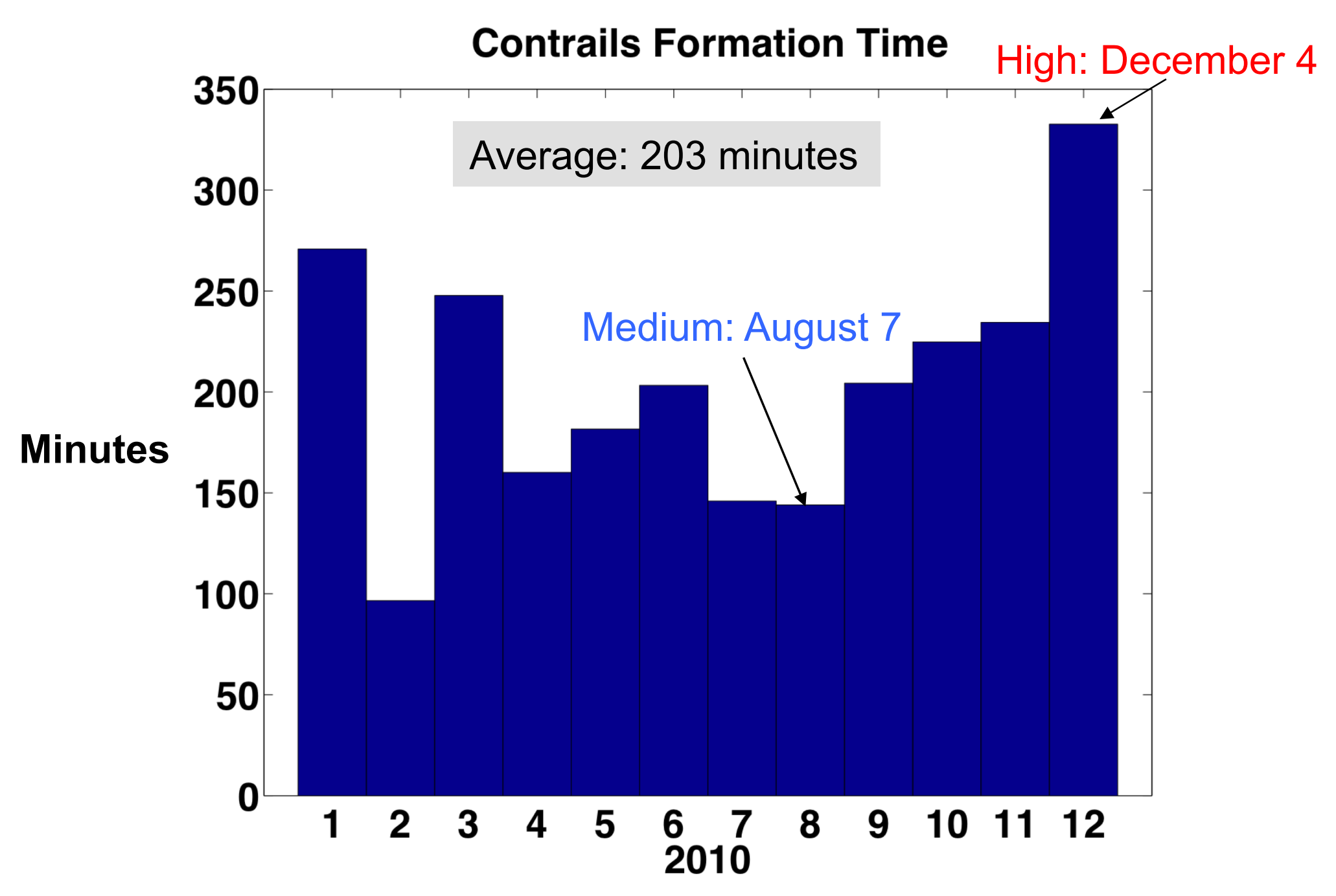




\section{Fuel Burn and GWP for Cross-Polar Trajectories}

\begin{tabular}{|ccccc|}
\hline \multirow{3}{*}{$8 / 7 / 10$} & FP & $\begin{array}{c}\text { Fuel } \\
\text { Burn } \\
\text { (ton) }\end{array}$ & $\begin{array}{c}\text { GWP } \\
(\mathbf{1 0 0 0 )}\end{array}$ & $\begin{array}{c}\text { Fuel } \\
\text { Savings } \\
(\%)\end{array}$ \\
\cline { 2 - 5 } & 91.7 & 442 & 0.0 \\
\cline { 2 - 5 } & GC & 89.1 & 430 & 2.8 \\
\hline WO & 84.4 & 407 & 8.0 \\
\hline \multirow{2}{*}{$12 / 4 / 10$} & FP & 91.4 & 441 & 0.0 \\
\cline { 2 - 5 } & GC & 89.3 & 431 & 2.3 \\
\cline { 2 - 5 } & WO & 87.4 & 422 & 4.4 \\
\hline
\end{tabular}

- Climate impact of cross-polar trajectories equivalent to about 400 tons of $\mathrm{CO}_{2}$ emissions

- WO trajectories reduce average fuel burn (GWP) by $8.0 \%$ and $4.4 \%$

- Conducting similar fuel savings analysis for Pacific Project Paper Trial and Data Analysis (Routes through Anchorage Center) 


\section{Cross-Polar Trajectories Generation}

- For each day (High: December 4, Medium: August 7)

For each Origin-Destination pair (15 pairs)

For each possible flight level (6 levels between $300-400$ )

Compute 1 flight plan trajectory

Compute 1 great circle trajectory

Compute 1 wind optimal trajectory

Compute 20 contrails avoidance trajectories

Compute fuel burn, emissions, GWP, persistent contrails formation time for each of the 23 trajectories 


\section{Trade-off between extra GWP and Contrails}

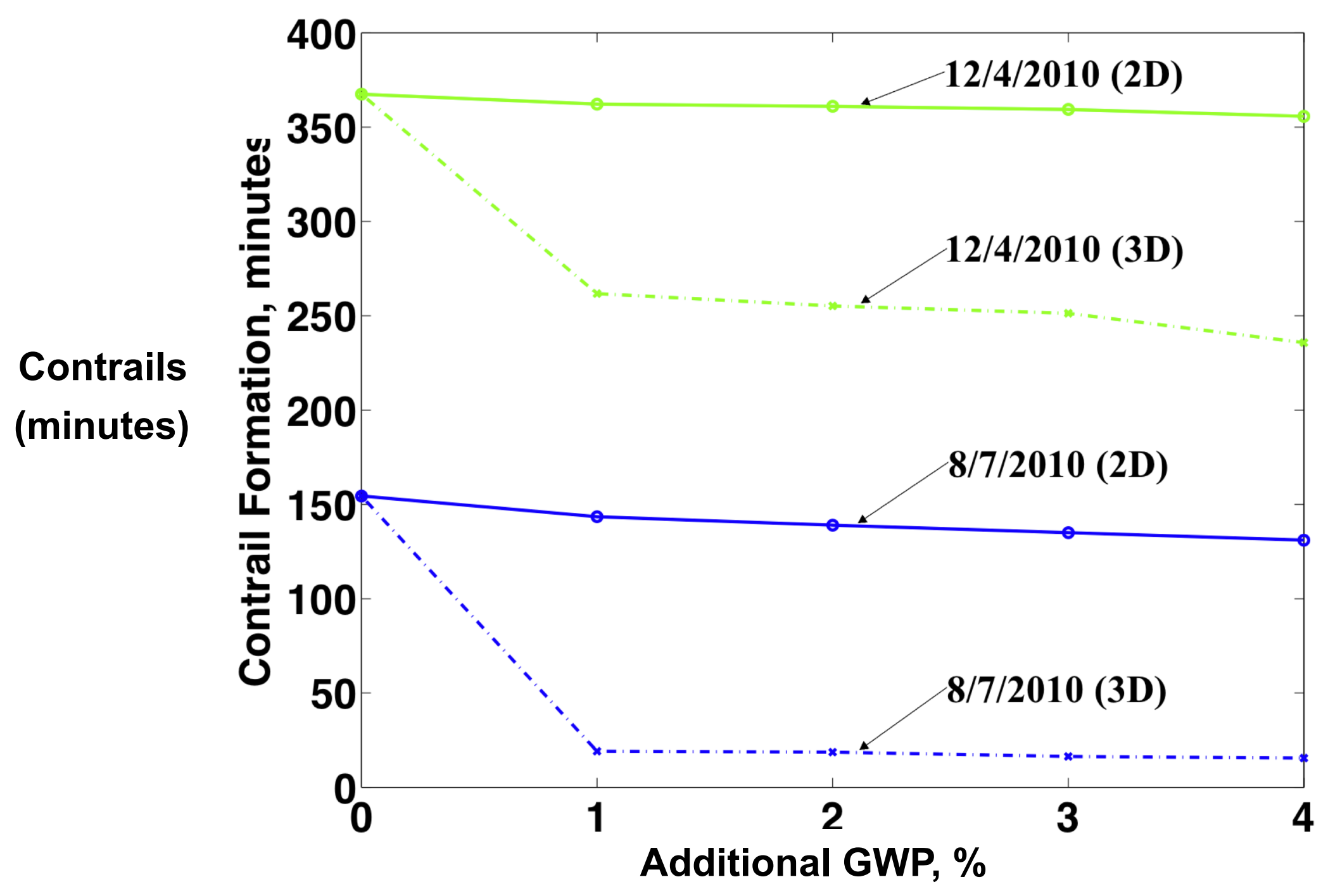




\section{Aggregate Global Temperature Potential (AGTP)}

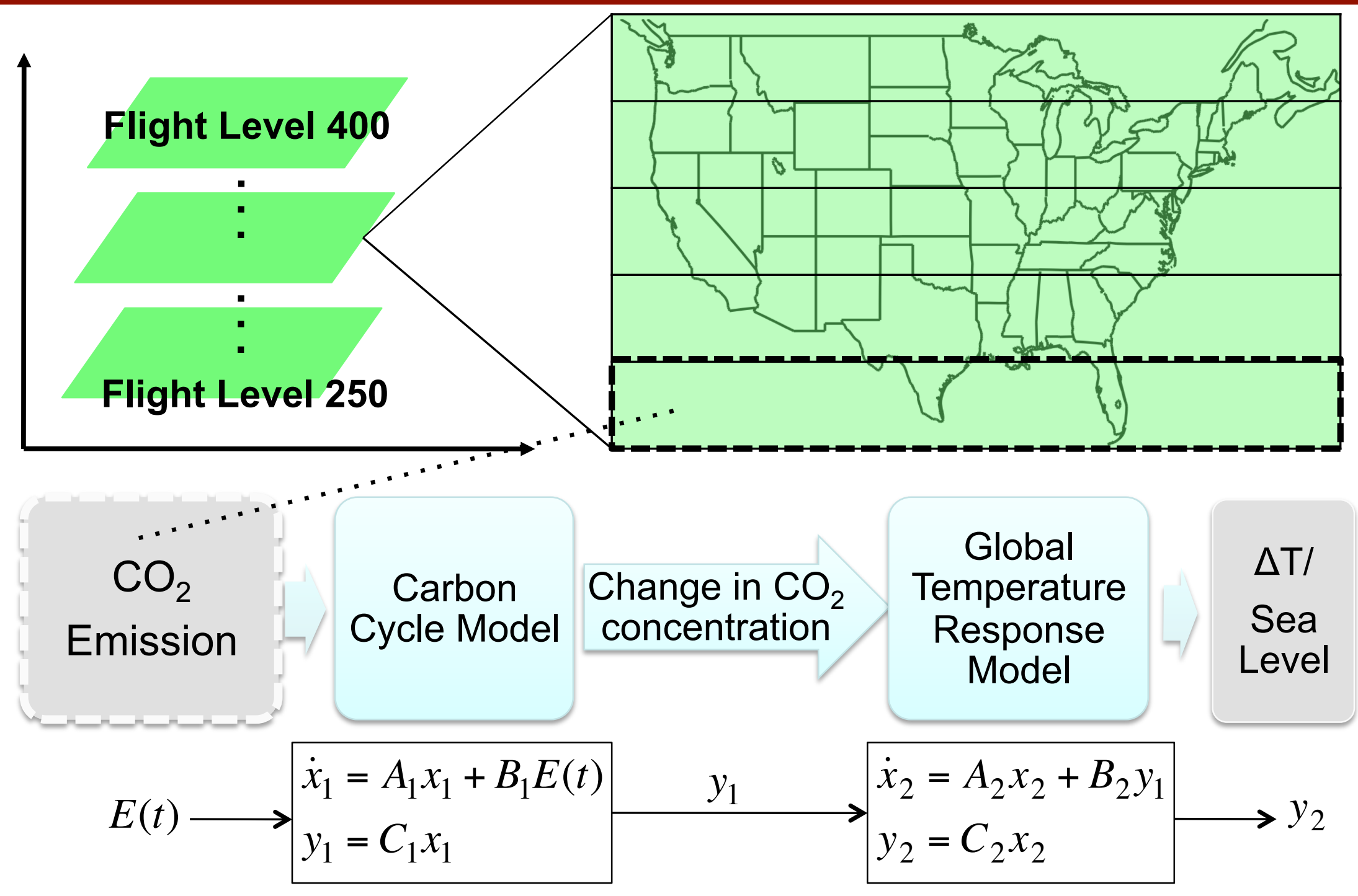




\section{Aggregate Global Temperature Potential (AGTP)}

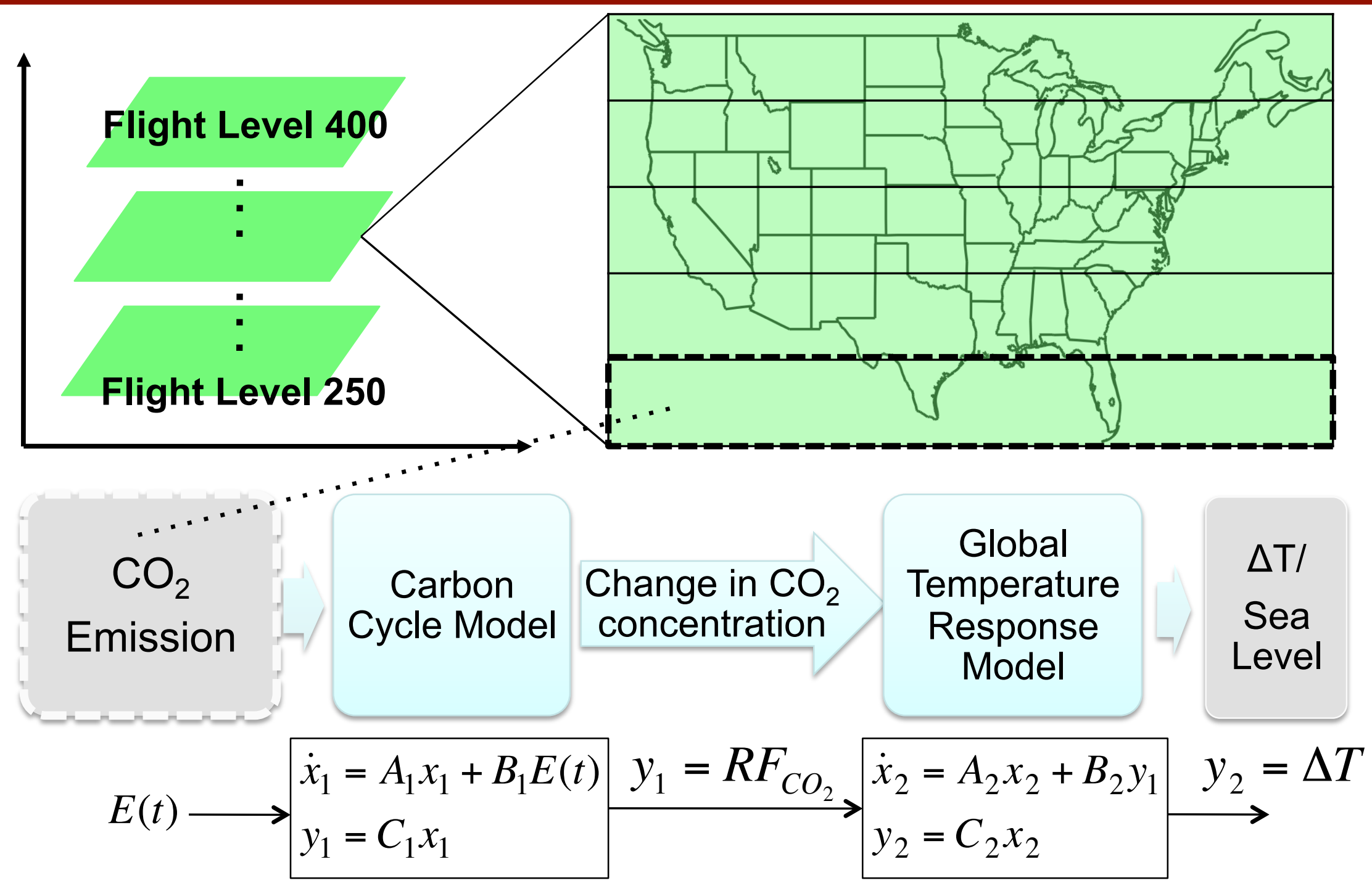




\section{Aggregate Global Temperature Potential (AGTP) Influence of emission and contrail parameters}
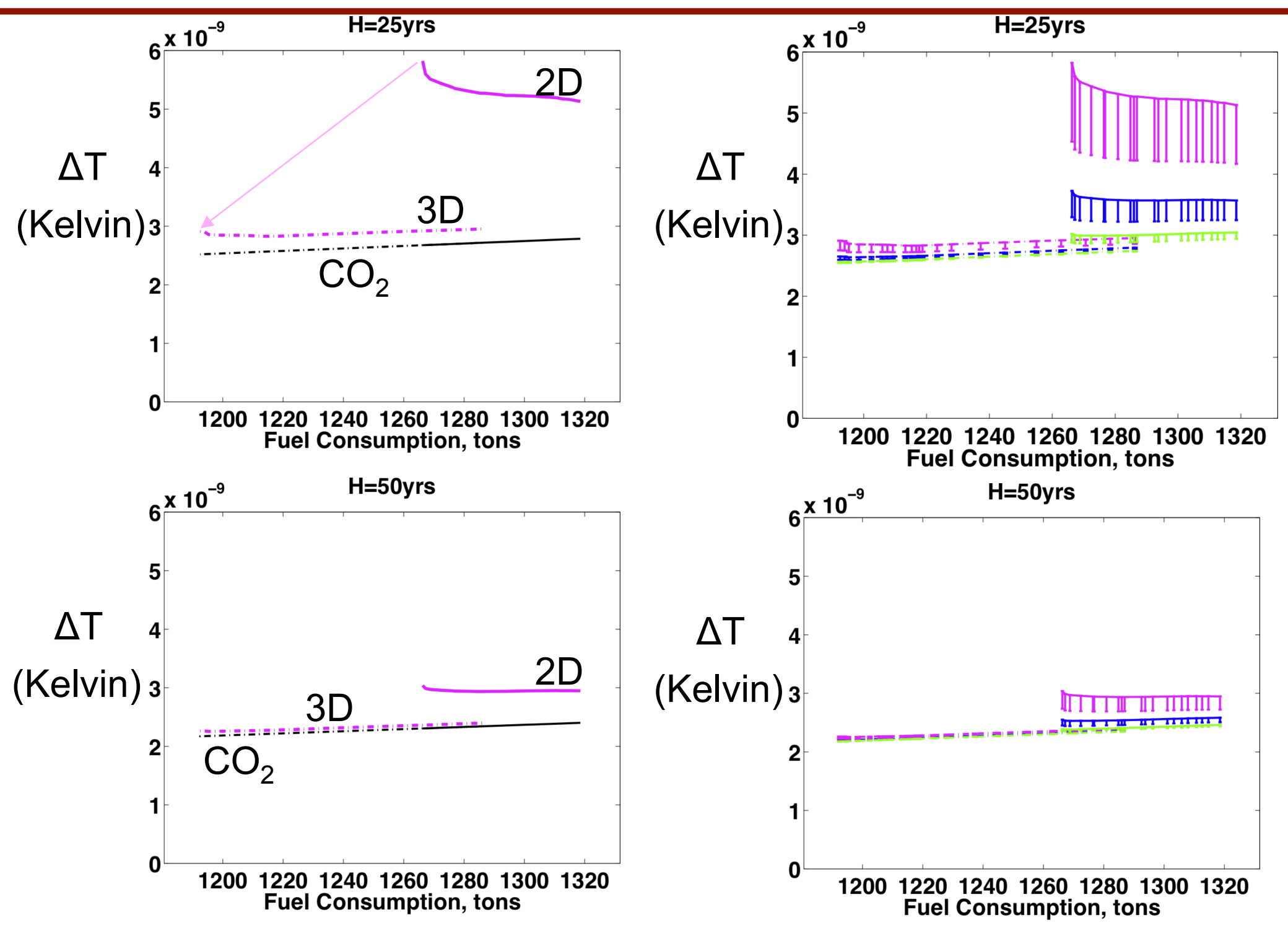


\section{Concluding Remarks}

- Developed an algorithm to calculate route optimization trajectories for aircraft while avoiding the regions of airspace that facilitate persistent contrails formation, convective weather or other hazards

- Presented trajectory optimization results for 15 origin-destination pairs between major international airports in the United States and Asia during the days with medium and high contrail formation in 2010

- Integrated contrails, emission models and optimized routing strategies to a national level airspace simulation with capability to visualize, evaluate technology and alternate operational concepts and provide inputs for policy-analysis tools to reduce the impact of aviation on the environment 